\title{
Effect of Psychosocial Distress on the Rate of Kidney Function Decline
}

\author{
Jae Young Kim, M.D. ${ }^{1}$, Young Su Joo, M.D. ${ }^{1,2}$, Jong Hyun Jhee, M.D., Ph.D. ${ }^{1,3}$, \\ Seung Hyeok Han, M.D., Ph.D. ', Tae-Hyun Yoo, M.D., Ph.D. ', \\ Shin-Wook Kang, M.D., Ph.D. ${ }^{7}$, and Jung Tak Park, M.D., Ph.D. '] \\ 'Department of Internal Medicine, College of Medicine, Institute of Kidney Disease Research, Yonsei University, Seoul, Republic of Korea; ${ }^{2}$ Division \\ of Nephrology, Department of Internal Medicine, Myongji Hospital, Goyang, Gyeonggi-do, Republic of Korea; ${ }^{3}$ Division of Nephrology, \\ Department of Internal Medicine, Gangnam Severance Hospital, Yonsei University College of Medicine, Seoul, Republic of Korea.
}

BACKGROUNDS: Chronic kidney disease is a growing global health problem. Psychosocial stress has been found to induce changes in biological processes and behavioral patterns that increase risks of cardiovascular and metabolic diseases. However, the association between psychosocial stress and kidney function is not well understood.

OBJECTIVE: To evaluate the association between psychosocial stress and kidney function decline.

DESIGN: In this prospective cohort study, psychosocial distress was assessed using the psychosocial well-being index short-form (PWI-SF).

PARTICIPANTS: Data of a total of 7246 participants were retrieved from a community-based cohort (Korean Genome and Epidemiology Study).

MAIN MEASURES: The rate of estimated glomerular filtration rate (eGFR) decline was calculated for each individual. Rapid eGFR decline was defined as a decrease of $\geq$ $3 \mathrm{~mL} / \mathrm{min} / 1.73 \mathrm{~m}^{2}$ per year. The presence of kidney disease was defined as eGFR $<60 \mathrm{~mL} / \mathrm{min} / 1.73 \mathrm{~m}^{2}$ at baseline or proteinuria of higher than trace levels from two consecutive urine test results.

KEY RESULTS: A total of 7246 participants were analyzed. The mean eGFR was $92.1 \pm 14.0 \mathrm{~mL} / \mathrm{min} / 1.73 \mathrm{~m}^{2}$. Rapid eGFR decline was observed in 941 (13.0\%) participants during a median follow-up of 11.7 years. When the participants were categorized into tertiles according to PWI-SF score, rapid eGFR decline was more prevalent in the group with the highest PWI-SF score (15.8\%) than in the group with the lowest score (12.2\%). Multivariate logistic regression analysis revealed that the risk of rapid eGFR decline was significantly increased in the tertile group with the highest PWI-SF score compared to the lowest group (odds ratio, 1.35; 95\% confidence interval, 1.15-1.59). This association was maintained even after adjusting for confounding variables and excluding participants with kidney disease.

CONCLUSIONS: Higher levels of psychosocial distress were closely associated with an increased risk of rapid kidney function decline.

Received August 10, 2020

Accepted December 29, 2020

Published online January 19, 2021
KEY WORDS: psychosocial distress; kidney disease; eGFR.

J Gen Intern Med 36(10):2966-74

DOI: $10.1007 / \mathrm{s} 11606-020-06573-9$

(C) Society of General Internal Medicine 2021

\section{INTRODUCTION}

Chronic kidney disease (CKD) is a growing global health problem. The prevalence of CKD is increasing despite controlling for the two major factors affecting kidney function, hypertension and diabetes. ${ }^{1}$ Because established CKD is irrevocable, identifying adjustable risk factors and changing the lifestyle that leads to CKD development are essential for reducing the CKD burden.

The interconnection of psychological and social factors with physical outcomes is present in several disease states. ${ }^{2-}$ ${ }^{4}$ Chronic psychological stress is closely related to the increased risk of incident coronary heart disease and poor cardiovascular prognosis. ${ }^{5-7}$ In addition, a recent meta-analysis revealed that the risk of acute coronary syndrome increased more than 2-fold after an acute episode of anger, stress, or depression. ${ }^{8}$ Stress-related psychosocial factors, such as chronic stress, life events, stress-prone personality, and poor social support, were found to be associated with higher cancer incidence in an otherwise healthy population. ${ }^{9}$ Among those who were already diagnosed with cancer, stress-related psychosocial factors were related with poor survival. Cardiovascular disease and CKD share common risk factors. Therefore, psychosocial health can potentially have a significant effect on kidney function. Nonetheless, evidence demonstrating such associations is limited.

Psychosocial health can be viewed as a complex interaction of emotional and social well-being. The social environment in which an individual resides also has consequential effects on the individual psychological well-being. Therefore, in this study, psychosocial stress was evaluated through measurements that encompassed various aspects of psychosocial health and its effect on kidney function was investigated. This was done by analyzing data from a prospective communitybased cohort. 


\section{METHODS}

\section{Study Population}

Data were retrieved from the Korean Genome and Epidemiology Study (KoGES), a prospective community-based cohort study. The purpose of KoGES was to investigate the genetic and environmental causes of common diseases in Koreans. Detailed methods and profiles on the development of KoGES have been described previously. ${ }^{10}$ The cohort consisted of 10,030 participants aged 40 to 69 years who were residents of Ansan (urban area) or Ansung (rural area), Korea. The participants underwent health examinations and replied to various surveys at baseline. Health examinations and surveys were followed up biennially from 2001 to 2014. The participants who completed the surveys on psychosocial distress were initially screened. Those with less than two values of serum creatinine were also excluded, taking into account the minimum data requirements for calculating changes in kidney function. A total of 7246 subjects were included in the final analysis (Fig. 1). The characteristics between those with and without baseline psychosocial distress data are presented in Supplemental Appendix 1.

All participants were voluntarily enrolled in the study and gave written informed consent. The study conforms to the ethical guidelines of the Declaration of Helsinki and was approved by the institutional review board of Yonsei University Health System Clinical Trial Center (Process No. 4-2016-0746).

\section{Data Collection}

Detailed description on data collection is provided in Supplemental Appendix 2. Data on demographic and socioeconomic status including health and lifestyle, anthropometric parameters, and medical history and receiving medications were collected. Blood pressure was measured by trained nurses using a mercury sphygmomanometer in the sitting position after at least a 5-min rest. The participants with a history of hypertension, with a blood pressure of $>140 / 90 \mathrm{mmHg}$, or receiving anti-hypertensive medications were considered hypertensive. The participants with a history of diabetes, blood glucose levels of $\geq 126 \mathrm{mg} / \mathrm{dL}$ after 8 -h fasting, postload glucose levels of $\geq 200 \mathrm{mg} / \mathrm{dL}$ in the 75 -g oral glucose tolerance test, or hemoglobin $\mathrm{A} 1 \mathrm{c}(\mathrm{HbA} 1 \mathrm{c}) \geq 6.5 \%$ or those receiving oral medication and/or insulin treatment for hyperglycemia were considered diabetic. Laboratory data were collected at baseline and at consecutive visits. Serum creatinine levels were measured using the Jaffé method and were reduced by a calibration factor of $5 \%$ for standardization to isotope dilution mass spectrometry reference method values. ${ }^{11}$ Estimated glomerular filtration rate (eGFR) was calculated using the CKD epidemiology collaboration equation. ${ }^{12}$ First-void urine samples were collected and analyzed using URISCAN Pro II (YD Diagnostics Corp., Seoul, Korea). The degree of proteinuria scored as absent, trace, $1+, 2+$, or $3+$ according to a color scale in the urine test strips correlated with urine protein levels of $<10,10-20,>30,>100$, and $>500 \mathrm{mg} / \mathrm{dL}$, respectively. ${ }^{13}$ The presence of kidney disease was defined as eGFR $<60 \mathrm{~mL} / \mathrm{min} / 1.73 \mathrm{~m}^{2}$ at baseline or proteinuria of higher than trace levels from two consecutive urine test results.

\section{Assessment of Psychosocial Distress}

The level of psychosocial distress was measured using the psychosocial well-being index short-form (PWI-SF) survey. The PWI-SF contains 18 items and evaluates social performance and self-confidence (eight items), depression (three items), sleep disturbances and anxiety (three items), and overall well-being and vitality (four items). Each item ranges from 0 ("strongly disagree") to 3 ("strongly agree") based on a 4point Likert scale. The total score ranges from 0 to 54 points. The PWI-SF has been used to assess the levels of psychosocial distress in different populations, including patients with stroke, industry workers, and firefighters. ${ }^{14-18}$ The validation and reliability of the PWI-SF have been previously demonstrated. ${ }^{19}$ The participants were required to answer questions on changes in mood, emotions, and behaviors over the last few weeks and were classified according to the total PWI-SF score. Detailed descriptions and a copy of the PWI-SF are provided in the Supplemental Appendix 3.

\section{Outcome Measures}

The annual eGFR decline for each participant was calculated using linear least-squares regression models, as previously reported. ${ }^{20,}{ }^{21}$ Individual eGFR measurements during the follow-up period were included in the models. To estimate the annual decrease in eGFR, the regression line closest to the patient's eGFR measurement was determined so that the sum of the squared deviations of the individual eGFR measurements from the line is minimized. Annual eGFR decline was defined as the slope of the least-squares regression line of the eGFR vs. time for each patient and expressed as $\mathrm{mL} / \mathrm{min} / 1.73$ $\mathrm{m}^{2}$ per year. Rapid renal function decline was defined as an annual decrease of $>3 \mathrm{~mL} / \mathrm{min} / 1.73 \mathrm{~m}^{2}$ per year based on the definition used in previous investigations. ${ }^{20,} 22$

\section{Statistical Analysis}

Statistical analysis was performed using SAS software version 9.4 (SAS Institute Inc., Cary, NC, USA), and STATA version 14.2 (Stata Corp, TX, USA). Continuous variables were expressed as means \pm standard deviations or median and interquartile range, and categorical variables were expressed as absolute numbers and percentages. The normality of distribution was ascertained using the Kolmogorov-Smirnov test. Normally distributed continuous variables between groups were compared by analysis of variance and Tukey honest significant difference post hoc test. Categorical variables were examined using the chi-square test or Fisher's exact test. Study participants were stratified into PWI-SF score tertiles for analysis. Additionally, previously proposed cutoff values for the 


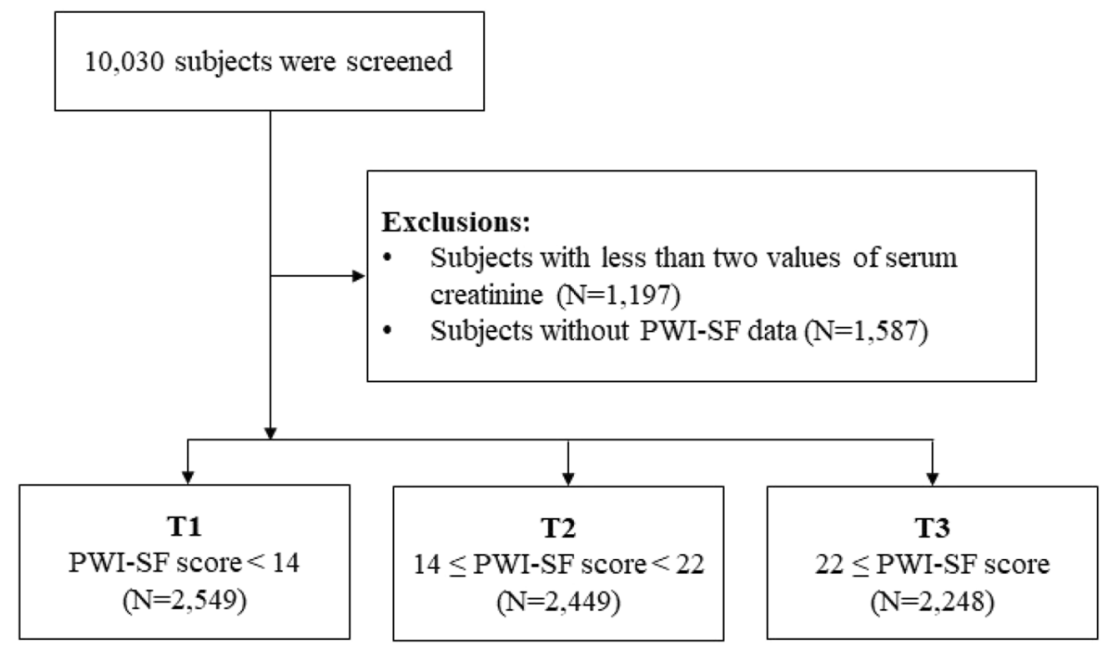

Figure 1 Flowchart of study participants. PWI-SF, psychosocial well-being index short form.

level of stress were also used for a sensitivity analysis; healthy group (PWI-SF score $\leq 8$ ), latent stress group (PWI-SF score 9-26), and stress group (PWI-SF score $\geq 27$ ). ${ }^{18,19}$ Multivariable logistic regression analysis was performed to evaluate the relationship between psychosocial distress and rapid eGFR decline. Variables with statistical significance in univariate logistic regression analyses and variables known to affect kidney function were included in the adjusted models. Model 1 was not adjusted for confounding variables. Model 2 was adjusted for age, sex, and baseline eGFR. Model 3 included demographic factors and comorbidities including body mass index (BMI), level of income, marital status, smoking, energy intake, systolic blood pressure, history of hypertension, and diabetes mellitus. Model 4 was further adjusted for laboratory data which included total cholesterol and high-sensitivity $\mathrm{C}$ reactive protein (hsCRP). Restricted cubic spline analysis was conducted with PWI-SF scores as continuous variables to test the non-linear relationship between psychosocial distress and rapid eGFR decline. This was performed by eliminating each 2.5 percentile of the upper and lower PWI-SF scores to reduce distortion. $p$ values $<0.05$ were considered statistically significant.

\section{RESULTS}

\section{Baseline Characteristics}

The baseline characteristics of the study subjects are shown in Table 1 . The mean age was $52.3 \pm 8.8$ years, and $47.8 \%$ of the patients were men. The mean eGFR was $92.1 \pm 14.0 \mathrm{~mL} / \mathrm{min} /$ $1.73 \mathrm{~m}^{2}$. The average PWI-SF score was $17.3 \pm 8.7$. In 7173 (99.0\%) participants, the number of follow-up visits was $\geq 3$ during the study period (Supplemental Appendix 4).

When stratified into PWI-SF score tertiles, the participants in the higher score group tended to have lower education and income and were more likely to be divorced. With regard to laboratory parameters, eGFR and the serum levels of albumin, total cholesterol, and
hsCRP were similar between the tertile groups. The prevalence of hypertension and diabetes increased linearly as the PWI-SF score increased.

\section{Annual eGFR Decline and Psychosocial Distress Levels}

During a median follow-up of 11.7 years (range, 3.5-12.7 years), the overall mean eGFR decline was $1.74 \pm 1.71 \mathrm{~mL} /$ $\mathrm{min} / 1.73 \mathrm{~m}^{2}$ per year. The comparison of the annual eGFR decline among the tertiles indicated that the decline was faster in the third tertile than in the first and second tertiles. However, the difference in the annual eGFR decline between the first and second tertiles was not significant (Table 2).

\section{Rapid eGFR Decline}

Rapid eGFR decline, defined as an eGFR decline of $>3 \mathrm{~mL} / \mathrm{min} /$ $1.72 \mathrm{~m}^{2}$ per year, was observed in $941(13.0 \%)$ participants. The decline was significantly more prevalent in the tertile with the highest PWI-SF score than in the first and second tertiles $(p<$ 0.001 , Table 2).

\section{Impact of Psychosocial Distress on Rapid eGFR Decline}

The risk of rapid eGFR decline was stratified among the tertiles using multivariable logistic regression models (Table 3 ). The analysis showed that the unadjusted odds ratio (OR) significantly increased in subjects in the highest tertile group compared to those in the lowest tertile group (model 1, OR, 1.35; $95 \%$ confidence interval $[\mathrm{CI}], 1.15-1.59 ; p<0.001)$. This association remained significant after adjusting for confounding variables (model 4, OR, 1.30; 95\% CI, 1.08-1.56; $p=0.005$ ). The association between rapid eGFR decline and PSW-SF scores, treated as a continuous variable, was also evaluated (Table 3). Each standard deviation increase in the PWI-SF score was associated with a $16 \%$ increase in the risk of rapid eGFR decline (model 1, OR, 1.16; 95\% CI, 1.09-1.24; $p<0.001$ ). The 
Table 1 Baseline Characteristics Among Study Groups Stratified by the Level of Psychosocial Distress

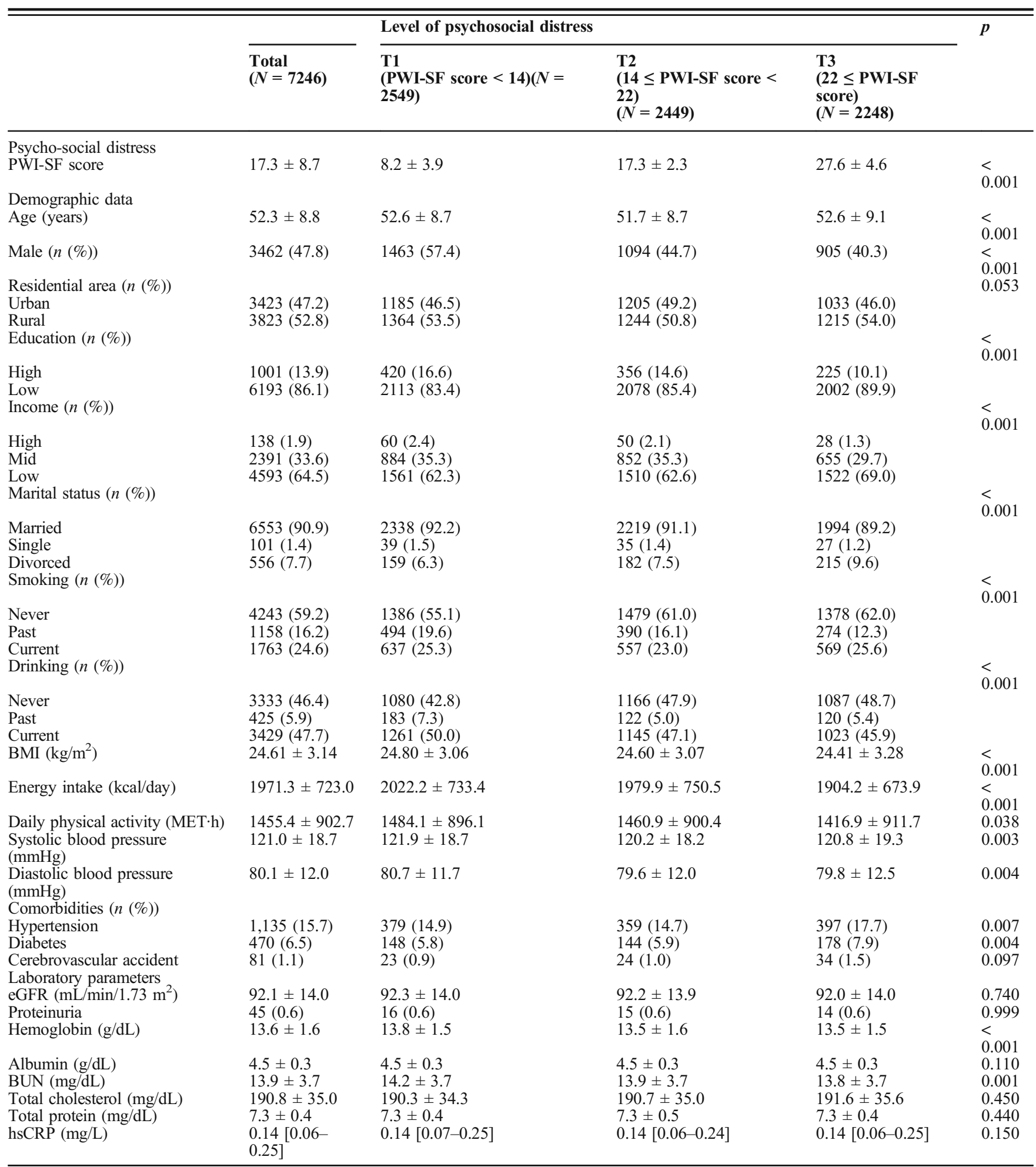

PWI-SF, psychosocial well-being index short-form; BMI, body mass index; MET, metabolic equivalent; eGFR, estimated glomerular filtration rate; $B U N$, blood urea nitrogen; hsCRP, high-sensitivity C-reactive protein

Data are presented as mean $\pm S D$, median [interquartile range], or $n(\%)$

relationship between rapid eGFR decline and PWI-SF score was robust even after adjusting for confounders, including comorbidities and socioeconomic status (model 4, OR, 1.13; $95 \%$ CI, $1.05-1.22 ; p=0.001)$.
When the association between PWI-SF score and annual eGFR decline, both as continuous variables, was evaluated, a significant inverse relationship $(\gamma=-0.052, p<0.001)$ was found. 
Table 2 Renal Function Decline Rate Among Groups Stratified by the Level of Psychosocial Distress

\begin{tabular}{|c|c|c|c|c|c|}
\hline & \multirow[t]{2}{*}{ Total } & \multicolumn{3}{|c|}{$\begin{array}{l}\text { Level of psychosocial } \\
\text { distress }\end{array}$} & \multirow[t]{2}{*}{$p$} \\
\hline & & T1 & $\mathbf{T} 2$ & T3 & \\
\hline $\begin{array}{l}\text { Renal function } \\
\text { decline rate }(\mathrm{mL} / \\
\text { min } / 1.73 \mathrm{~m}^{2} / \\
\text { year })\end{array}$ & $\begin{array}{l}-1.74 \\
\pm 1.71\end{array}$ & $\begin{array}{l}-1.71 \\
\pm 1.60\end{array}$ & $\begin{array}{l}-1.63 \\
\pm 1.58\end{array}$ & $\begin{array}{l}-1.88 \\
\pm 1.94\end{array}$ & $<.001^{*}$ \\
\hline $\begin{array}{l}\text { Rapid renal } \\
\text { function decline } \\
(n(\%))\end{array}$ & $\begin{array}{l}941 \\
(13.0)\end{array}$ & $\begin{array}{l}311 \\
(12.2)\end{array}$ & $\begin{array}{l}275 \\
(11.2)\end{array}$ & $\begin{array}{l}355 \\
(15.8)\end{array}$ & $\begin{array}{l}< \\
0.001\end{array}$ \\
\hline
\end{tabular}

PWI-SF, psychosocial well-being index; eGFR, estimated glomerular filtration rate

All continuous variables are expressed as mean $\pm S D$, or absolute values (percentage). Rapid renal function decline was defined as an eGFR decrease of $>3 \mathrm{~mL} / \mathrm{min} / 1.73 \mathrm{~m}^{2}$ per year

$* p<0.001$ by analysis of variance and Tukey post hoc test. $p$ values were smaller than 0.05 for all interactions except $T 1$ vs. T2

Since multivariable logistic regression models revealed a non-linear association between psychosocial distress and the risk of rapid eGFR decline, restrictive cubic spline analysis was conducted. There was a significant and gradual increment in the OR for rapid eGFR decline as the PWI-SF score increased (Fig. 2).

\section{Subgroup Analysis}

The relationship between psychosocial distress and rapid eGFR decline was further investigated in subgroups stratified by age ( $<55$ or $\geq 55$ years), sex (male or female), level of education (primary/secondary or higher), hypertension (presence or absence), and diabetes (presence or absence). There were no significant interactions in any of the subgroups, indicating that the relationship between psychosocial distress and the risk of rapid eGFR decline was consistently significant across these subgroups (Fig. 3).

\section{Sensitivity Analysis}

Subsequent evaluations were performed for sensitivity analyses. Analyses performed after excluding a total of 33 participants with pre-existing mental disorders of which the specific diagnosis was uninvestigated (model 4, OR, 1.30; 95\% CI, $1.09-1.56 ; p=0.004$ ) (Supplemental Appendix 5), excluding current smokers (model 4, OR, 1.37; 95\% CI, 1.10-1.69; $p=$ 0.004) (Supplemental Appendix 6), excluding 179 participants with the presence of kidney diseases (model 4, OR, $1.29 ; 95 \%$ CI, 1.07-1.55; $p=0.007$ ) (Supplemental Appendix 7), and excluding those with less than three follow-up visits (model 4, OR, 1.31; 95\% CI, 1.09-1.58; $p=$ 0.004) (Supplemental Appendix 8) all showed that the risk of rapid kidney function decline was significantly elevated in the highest PWI-SF tertile than the lowest. Evaluations using different PWI-SF cutoff points were also performed. When the lowest and middle tertile groups were combined, the risk of rapid kidney function decline was significantly increased in the highest tertile group when compared to the combined group (model 4, OR, 1.35; 95\% CI, $1.15-1.58 ; p<0.001$ ) (Supplemental Appendix 9). In addition, compared to the healthy group (PWI-SF score $\leq 8$ ), the stress group (PWI-SF score $\geq 27$ ) was associated with an elevation of the risk for rapid kidney function decline (model 4, OR, 1.34; 95\% CI, 1.04-1.74; $p=0.024$ ) (Supplemental Appendix 10).

\section{DISCUSSION}

The kidney function deteriorated more quickly in individuals with higher levels of psychosocial distress. In addition, higher PWI-SF scores, which estimate the level of psychosocial distress, were significantly associated with higher risks of rapid eGFR decline, and this association was independent of underlying comorbidities and sociodemographic factors.

Psychosocial distress has been proposed to be linked to the development of hypertension and metabolic derangements such as type 2 diabetes or metabolic syndrome in several studies. ${ }^{23-27}$ Since hypertension and diabetes are the two most well-known risk factors for $\mathrm{CKD},{ }^{28}$ there is a high probability that the effect of psychosocial distress on kidney function observed in this study could have been mediated by these two factors. This notion was supported by the finding that the participants in the group with higher levels of psychosocial

Table 3 Multivariate Models of the Risk of Rapid Renal Function Decline According to Psychosocial Distress Level

\begin{tabular}{|c|c|c|c|c|c|c|c|c|}
\hline & \multicolumn{2}{|l|}{ Model 1} & \multicolumn{2}{|l|}{ Model 2} & \multicolumn{2}{|l|}{ Model 3} & \multicolumn{2}{|l|}{ Model 4} \\
\hline & OR $(95 \%$ CI $)$ & $p$ & OR $(95 \%$ CI $)$ & $p$ & OR $(95 \% \mathrm{CI})$ & $p$ & OR $(95 \%$ CI) & $p$ \\
\hline PWI-SF score/SD & $\begin{array}{l}1.16(1.09-1.24) \\
\text { V PUI-SF score }\end{array}$ & $<0.001$ & $1.16(1.08-1.24)$ & $<0.001$ & $1.13(1.05-1.22)$ & 0.001 & $1.13(1.05-1.22)$ & 0.001 \\
\hline \multicolumn{9}{|c|}{ Tertiles categorized by PWI-SF score } \\
\hline $\mathrm{T} 2$ & $0.91(0.77-1.08)$ & 0.288 & $0.94(0.79-1.12)$ & 0.501 & $0.93(0.77-1.12)$ & 0.435 & $0.92(0.76-1.11)$ & 0.396 \\
\hline $\mathrm{T} 3$ & $1.35(1.15-1.59)$ & $<0.001$ & $1.36(1.15-1.62)$ & $<0.001$ & $1.30(1.08-1.56)$ & 0.005 & $1.30(1.08-1.56)$ & 0.005 \\
\hline
\end{tabular}

PWI-SF, psychosocial well-being index short-form; SD, standard deviation; OR, odds ratio; CI, confidence interval; eGFR, estimated glomerular filtration rate; BMI, body mass index; hsCRP, high-sensitivity C-reactive protein

Rapid renal function decline was defined as $3 \mathrm{~mL} / \mathrm{min} / 1.73 \mathrm{~m}^{2}$ per year or higher during follow-up

Model 1 was unadjusted

Model 2 was adjusted for age, sex, and eGFR

Model 3 was adjusted for BMI, level of income, marital status, smoking, energy intake, systolic blood pressure, hypertension, and diabetes mellitus, and included the variables from model 2

Model 4 was adjusted for total cholesterol and hsCRP, and included the variables from model 3 


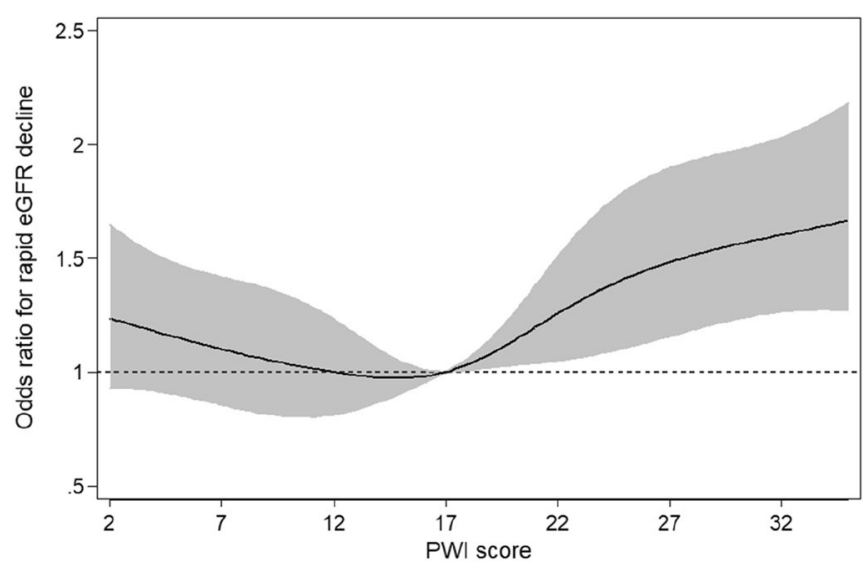

Figure 2 Restricted cubic spline curve for the association between psychosocial distress levels and rapid renal function decline. Adjusted for age, sex, eGFR, BMI, level of income, marital status, smoking, energy intake, systolic blood pressure, hypertension, diabetes mellitus, total cholesterol, and hsCRP. The median PWI score was used as a reference. The gray area represents the 95\% confidence interval. eGFR, estimated glomerular filtration rate; BMI, body mass index; hsCRP, high-sensitivity C-reactive protein; PWI-SF, psychosocial well-being shortform.

distress were more likely to have hypertension or diabetes. However, baseline blood pressure did not show a clear relationship with psychosocial distress levels. This is probably because hypertensive patients were already receiving treatment for high blood pressure. Nonetheless, other mechanisms may play a role because the relationship between the levels of psychosocial distress and rapid kidney function decline remained significant even after adjusting for the presence of hypertension and diabetes.

Chronic inflammation is an important factor that can potentially initiate a variety of chronic diseases, including chronic kidney disease. ${ }^{29}$ In addition, inflammation has been suggested as a key pathway linking psychosocial distress and stress-related diseases. ${ }^{30}$ Therefore, it would be reasonable to assume inflammation to be a pivotal factor mediating psychosocial distress and kidney disease. The association between psychosocial distress and rapid kidney function decline found in the current study may also be intervened by chronic inflammation triggered by psychosocial distress conditions. However, the association between kidney function decline and psychosocial distress was significant even after adjustments were made for hsCRP. Although hsCRP levels do not fully represent the chronic inflammation status, this opens the possibility that factors other than inflammation may also play a role in relating the effects of psychosocial stress to kidney disease.

Previous investigations in animals have proposed a possible link between psychosocial distress and kidney disease. Studies have shown that kidney weight and volume and glomerular volume and density were significantly lower in prepubertal rats subjected to immobilization stress. ${ }^{31,32}$ Furthermore, the number of glomeruli per kidney was decreased in the stresschallenged group. However, direct evidence that stress is a risk factor for kidney disease in humans is limited.

Psychosocial distress usually results in undesirable behavioral patterns such as smoking, drug abuse, unhealthy diet, and low physical activity, which are potential risk factors for rapid kidney function decline. ${ }^{33,}{ }^{34}$ It is possible that the rapid decline in kidney function observed in individuals with higher levels of psychosocial distress could be due to unhealthy behavioral factors rather than the imposed stress itself. However, the relationship between rapid kidney function decline and psychosocial distress remained significant even after adjusting for behavioral factors. In addition, the results of sensitivity analysis excluding current smokers were similar to the overall findings, suggesting that other mechanisms might play a role. On the other hand, kidney function decline is known to be more rapid in subjects with prevalent CKD. ${ }^{35}$ Moreover, psychosocial factors, including depression and anxiety, are common in patients with CKD. ${ }^{36}$ Therefore, the association with rapid kidney function decline could be due to the underlying kidney disease rather than psychosocial distress. A clear cause-effect relationship cannot be established, given the observational nature of this study. However, the fact that the overall findings agreed with sensitivity analysis results excluding subjects with underlying kidney disease suggests that such bias is unlikely. Notwithstanding, further studies on the effect of stress modification on kidney function are needed.

This study has several strengths. First, data were obtained from a prospective cohort with up to 12 years of follow-up. Second, various confounding factors, including lifestyle and socioeconomic variables, were included in the analyses. Third, although clinical evidence directing psychosocial stress as an influencing factor of kidney disease has not yet been addressed in the literature, studies have shown associations between other diseases and some elements of psychosocial stressors. The risk of coronary heart disease has been shown to be increased in patients with anxiety disorder, ${ }^{37}$ and hypertensive patients have been examined to have more frequent episodes of anger. ${ }^{38}$ However, psychosocial stress consists of a variety of factors, including anger, anxiety, depression, stress, and social relationships, and few studies have evaluated the impact of psychosocial stress as a whole. In this regard, the current study is noteworthy in that it 


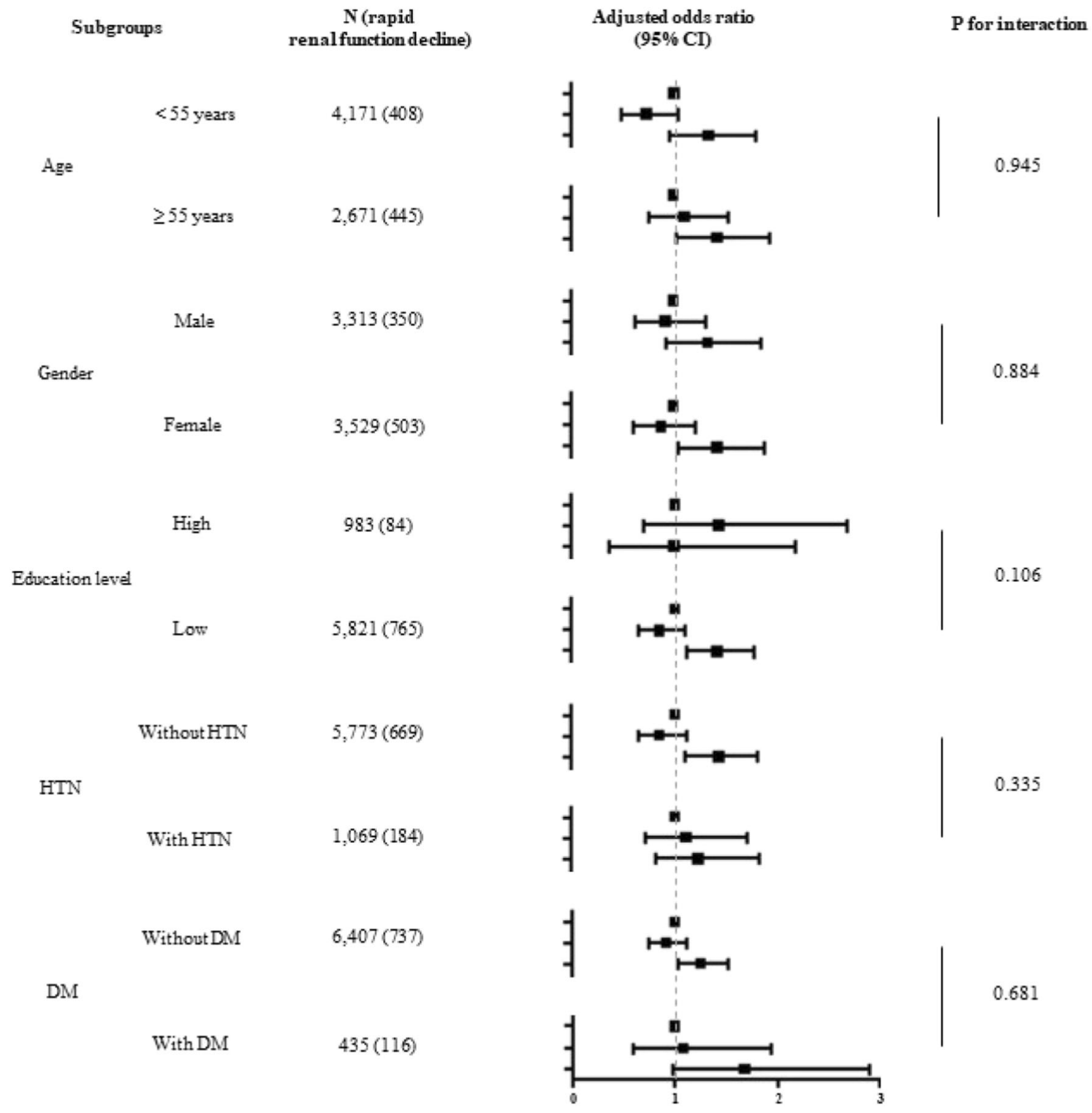

Figure 3 Forest plots for subgroup analysis. Adjusted for age, sex, eGFR, BMI, level of income, marital status, smoking, energy intake, systolic blood pressure, hypertension, diabetes mellitus, total cholesterol, and hsCRP. HTN, hypertension; DM, diabetes mellitus; eGFR, estimated glomerular filtration rate; BMI, body mass index; hsCRP, high-sensitivity C-reactive protein.

evaluates the effect of psychosocial stress using an index which embraces all of composing factors.

This study has several limitations. First, the limitations of the observational nature must be considered. The causality between stress and kidney function should be confirmed by future controlled experimental trials. Second, stress levels were measured only once. Although the current results suggest that increased stress levels at certain time points may affect future kidney function, it is difficult to determine the time-varying effect of changes in stress levels. Additional cohort studies with sequential stress level measurements are warranted. Third, the number of participants with missing PWI-SF data were relatively large (15.8\%). However, baseline kidney function as well as major risk factors for kidney diseases was comparable between those with and without PWI-SF data suggesting that the possibility of the missing data having effect on the key findings would not be large. Fourth, the lack of data on drugs, including renin-angiotensin-aldosterone system inhibitors and antidepressants, would have limited the thorough consideration of confounding factors.

In conclusion, in this community-based prospective cohort, higher levels of psychosocial distress were associated with an increased risk of rapid kidney function decline. The increase in risk was independent of sociodemographic characteristics or behavioral patterns. However, further studies with longitudinal data on psychosocial distress would be needed to confirm the findings of this study.

Supplementary Information The online version contains supplementary material available at https://doi.org/10.1007/s11606-02006573-9.

Acknowledgments: The epidemiological data used in this study were obtained from the Korean Genome and Epidemiology Study (4851-302) of the National Research Institute of Health, Centers for 
Disease Control and Prevention, Ministry for Health and Welfare, Republic of Korea.

Corresponding Author: Jung Tak Park, M.D., Ph.D.; Department of Internal Medicine, College of Medicine, Institute of Kidney Disease Research, Yonsei University, Seoul, Republic of Korea (e-mail: jtpark@yuhs.ac).

Compliance with Ethical Standards:

Conflict of Interest: The authors declare that they do not have a conflict of interest.

\section{REFERENCES}

1. USRDS. Annual data report: atlas of chronic kidney disease and endstage renal disease in the United States. National Institutes of Health, National Institute of Diabetes, Digestive and Kidney Diseases. 2013.

2. Robinson KL, McBeth J, MacFarlane GJ. Psychological distress and premature mortality in the general population: a prospective study. Ann Epidemiol 2004; 14(7):467-472.

3. May M, McCarron P, Stansfeld S, Ben-Shlomo Y, Gallacher J, Yarnell $\mathbf{J}$, et al. Does psychological distress predict the risk of ischemic stroke and transient ischemic attack? The Caerphilly StudyStroke 2002;33(1):712.

4. Yasuda N, Mino Y, Koda S, Ohara $\mathbf{H}$. The differential influence of distinct clusters of psychiatric symptoms, as assessed by the general health questionnaire, on cause of death in older persons living in a rural community of Japan. J Am Geriatr Soc 2002;50(2):313-320.

5. Stansfeld SA, Fuhrer R, Shipley MJ, Marmot MG. Psychological distress as a risk factor for coronary heart disease in the Whitehall Il Study. Int J Epidemiol 2002;31(1):248-255.

6. Yusuf S, Hawken S, Ônpuu S, Dans T, Avezum A, Lanas F, et al. Effect of potentially modifiable risk factors associated with myocardial infarction in 52 countries (the INTERHEART study): case-control study. Lancet. 2004;364(9438):937-952.

7. Steptoe A, Kivimäki M. Stress and cardiovascular disease. Nat Rev Cardiol 2012;9(6):360.

8. Edmondson D, Newman JD, Whang W, Davidson KW. Emotional triggers in myocardial infarction: do they matter? Eur Heart J 2012;34(4):300-306. https://doi.org/10.1093/eurheartj/ehs398.

9. Chida Y, Hamer M, Wardle J, Steptoe A. Do stress-related psychosocial factors contribute to cancer incidence and survival? Nat Clin Pract Oncol 2008;5(8):466-475. https://doi.org/10.1038/ncponc1134.

10. Kim Y, Han B-G, group tK. Cohort Profile: The Korean Genome and Epidemiology Study (KoGES) Consortium. Int $\mathrm{J}$ Epidemiol 2016;46(2):e20-e20. https://doi.org/10.1093/ije/dyv316.

11. Matsushita K, Mahmoodi BK, Woodward M, Emberson JR, Jafar TH, Jee SH, et al. Comparison of risk prediction using the CKD-EPI equation and the MDRD study equation for estimated glomerular filtration rate. Jama. 2012;307(18):1941-1951. https://doi.org/10.1001/jama.2012. 3954.

12. Levey AS, Stevens LA, Schmid CH, Zhang YL, Castro AF, Feldman HI, et al. A new equation to estimate glomerular filtration rate. Ann Intern Med 2009;150(9):604-612.

13. Lim D, Lee DY, Cho SH, Kim OZ, Cho SW, An SK, et al. Diagnostic accuracy of urine dipstick for proteinuria in older outpatients. Kidney Res Clin Pract 2014;33(4): 199-203. https://doi.org/10.1016/j.krcp.2014.10. 003.

14. Health Examinees Study G. The Health Examinees (HEXA) study: rationale, study design and baseline characteristics. Asian Pac J Cancer Prev 2015;16(4):1591-1597. https://doi.org/10.7314/apjcp.2015.16.4. 1591.

15. Chang WH, Sohn MK, Lee J, Kim DY, Lee S-G, Shin Y-I, et al. Korean Stroke Cohort for functioning and rehabilitation (KOSCO): study rationale and protocol of a multi-centre prospective cohort study. BMC Neurol 2015;15(1):42. https://doi.org/10.1186/s12883-015-0293-5.
16. Koh DH, Han SS, Kim HR, Chang SJ, Choi SH, Won JU, et al. Analysis of the role of social support on job stress and psychosocial stress in automobile factory workers using Structural Equation Model. Korean J Occup Environ Med 2005;17(2):79-84.

17. Lim DK, Baek KO, Chung IS, Lee MY. Factors Related to Sleep Disorders among Male Firefighters. Ann Occup Environ Med 2014;26:11. https://doi.org/10.1186/2052-4374-26-11.

18. Park B, Lee MH, Kong SY, Lee ES. Psychosocial Health of Disease-Free Breast Cancer Survivors Compared with Matched Non-cancer Controls. Cancer Res Treat 2019;51(1):178-186. https://doi.org/10.4143/crt. 2017.585.

19. Chang $\mathbf{S}$. Standardization of collection and measurement for heath data. Kyechukmunhwasa, Seoul 2000:121-159.

20. Rifkin DE, Shlipak MG, Katz R, Fried LF, Siscovick D, Chonchol M, et al. Rapid kidney function decline and mortality risk in older adults. Arch Intern Med 2008;168(20):2212-2218. https://doi.org/10.1001/ archinte.168.20.2212.

21. Looker HC, Mauer M, Saulnier P-J, Harder JL, Nair V, Boustany-Kari CM, et al. Changes in Albuminuria But Not GFR are Associated with Early Changes in Kidney Structure in Type 2 Diabetes. J Am Soc Nephrol 2019;30(6): 1049-1059. https://doi.org/10.1681/asn.2018111166.

22. Shlipak MG, Katz R, Kestenbaum B, Siscovick D, Fried L, Newman A, et al. Rapid decline of kidney function increases cardiovascular risk in the elderly. J Am Soc Nephrol 2009;20(12):2625-2630. https://doi.org/ 10.1681/asn.2009050546.

23. Bedi M, Varshney VP, Babbar R. Role of cardiovascular reactivity to mental stress in predicting future hypertension. Clin Exp Hypertens 2000;22(1):1-22. https://doi.org/10.1081/ceh-100100058.

24. Harris CW, Edwards JL, Baruch A, Riley WA, Pusser BE, Rejeski WJ, et al. Effects of mental stress on brachial artery flow-mediated vasodilation in healthy normal individuals. Am Heart J 2000;139(3):405-411. https://doi.org/10.1016/s0002-8703(00)90083-8.

25. Engum A. The role of depression and anxiety in onset of diabetes in a large population-based study. J Psychosom Res 2007;62(1):31-38. https://doi.org/10.1016/j.jpsychores.2006.07.009.

26. Räikkönen K, Matthews KA, Kuller LH. Depressive symptoms and stressful life events predict metabolic syndrome among middle-aged women: a comparison of World Health Organization, Adult Treatment Panel III, and International Diabetes Foundation definitions. Diabetes Care 2007;30(4):872-877. https://doi.org/10.2337/dc06-1857.

27. Novak M, Björck L, Giang KW, Heden-Ståhl C, Wilhelmsen L, Rosengren A. Perceived stress and incidence of Type 2 diabetes: a 35year follow-up study of middle-aged Swedish men. Diabet Med 2013;30(1):e8-16. https://doi.org/10.1111/dme.12037.

28. Levey AS, Coresh J, Balk E, Kausz AT, Levin A, Steffes MW, et a1. National Kidney Foundation practice guidelines for chronic kidney disease: evaluation, classification, and stratification. Ann Intern Med 2003;139(2): 137-147.

29. Silverstein DM. Inflammation in chronic kidney disease: role in the progression of renal and cardiovascular disease. Pediatr Nephrol 2009;24(8): 1445-1452. https://doi.org/10.1007/s00467-008-1046-0.

30. Liu YZ, Wang YX, Jiang CL. Inflammation: The Common Pathway of Stress-Related Diseases. Front Hum Neurosci 2017;11:316. https://doi. org/10.3389/fnhum.2017.00316.

31. Benchimol de Souza D, Silva D, Marinho Costa Silva C, Barcellos Sampaio FJ, Silva Costa W, Martins Cortez C. Effects of immobilization stress on kidneys of Wistar male rats: a morphometrical and stereological analysis. Kidney Blood Press Res 2011;34(6):424-429. https://doi.org/ 10.1159/000328331.

32. Marchon RG, Ribeiro CT, Costa WS, Sampaio FJB, Pereira-Sampaio MA, de Souza DB. Immediate and Late Effects of Stress on Kidneys of Prepubertal and Adult Rats. Kidney Blood Press Res 2018;43(6):19191926. https://doi.org/10.1159/000496004.

33. Marmot MG, Stansfeld S, Patel C, North F, Head J, White I, et al. Health inequalities among British civil servants: the Whitehall II study. Lancet. 1991;337(8754): 1387-1393.

34. Rod NH, Grønbaek M, Schnohr P, Prescott E, Kristensen T. Perceived stress as a risk factor for changes in health behaviour and cardiac risk profile: a longitudinal study. J Intern Med 2009;266(5):467-475. 
35. Webster AC, Nagler EV, Morton RL, Masson P. Chronic Kidney Disease. Lancet. 2017;389(10075):1238-1252. https://doi.org/10.1016/S01406736(16)32064-5.

36. Fischer MJ, Kimmel PL, Greene T, Gassman JJ, Wang X, Brooks DH, et al. Sociodemographic factors contribute to the depressive affect among African Americans with chronic kidney disease. Kidney Int. 2010;77(11):1010-1019. https://doi.org/10.1038/ki.2010.38.
37. Roest AM, Martens EJ, de Jonge P, Denollet J. Anxiety and risk of incident coronary heart disease: a meta-analysis. J Am Coll Cardiol 2010;56(1):38-46. https://doi.org/10.1016/j.jacc.2010.03.034.

38. Everson SA, Goldberg DE, Kaplan GA, Julkunen J, Salonen JT. Anger expression and incident hypertension. Psychosom Med 1998;60(6):730735. https://doi.org/10.1097/00006842-199811000-00014.

Publisher's Note: Springer Nature remains neutral with regard to jurisdictional claims in published maps and institutional affiliations. 